\title{
Accuracy of measuring the nematic order from intensity scatter: A simulation study
}

\author{
S. V. Savenko and Marjolein Dijkstra \\ Debye Institute, Soft Condensed Matter, Utrecht University, Princetonplein 5, 3584 CC Utrecht, The Netherlands
}

(Received 3 December 2003; published 27 July 2004)

\begin{abstract}
The determination of the nematic order parameter $S$ and the orientational distribution function (ODF) from scattering data involve severe approximations. The validity of these are studied here using Monte-Carlo simulations of hard spherocylinders with an aspect ratio of 15 for varying densities in the isotropic and nematic phase. The "exact" ODF of the rods, the "exact" value of $S$, and the intensity scatter $I(\vec{q})$ are determined directly in simulation. In addition, we determine the ODF and $S$ from the simulated intensity scatter which includes spatial and orientational correlations of the particles. We investigate whether correlations present in the interparticle scatter influences the determination of the single particle orientational distribution function by comparing the results obtained from scattering with the "exact" results measured directly in our simulations. We find that the nematic order parameter determined from the intensity scatter underestimates the actual value by $2-9 \%$. We also find that the values of $S$ and the ODF are insensitive to the absolute value of the scattering vector for $1.2 \pi<|\vec{q}| D<2 \pi$ which agrees well with the assumption proposed by Leadbetter that $I(q, \psi)$ along the equatorial arc is independent of $|\vec{q}|$. We also observe that the best fit of the "exact" ODF is given by the Maier-Saupe distribution when nematic director fluctuations are ignored, while the Gaussian distributions provides the best fit when these fluctuations are included.
\end{abstract}

DOI: 10.1103/PhysRevE.70.011705

\section{INTRODUCTION}

Liquids consisting of anisotropic molecules exhibit a number of liquid-crystalline phases, that are intermediate between the well-ordered crystalline solid and the isotropic fluid phase [see Fig. 1(a)]. The simplest liquid-crystal phase is the so-called nematic phase, in which the particles exhibit long-range orientational order but no translational order [see Fig. 1(b)]. Extensive theoretical and simulation studies are performed on hard-rod fluids, which serve as a good model for rodlike colloidal particles like the inorganic boehmite rods [1] or the biological TMV and $f d$ virus particles [2]. The phase behavior of colloidal hard rods starts with the seminal work of Onsager, who showed that infinitely thin hard rods exhibit an entropy-driven isotropic-nematic phase transition at sufficiently high densities [3]. Subsequently, extensive computer simulation studies showed that also finite hard rods show a nematic phase [4-7], a smectic phase [4-6,8] and a columnar phase [5]. More recently, the phase diagram of hard rods is determined as a function of the aspect ratio and density of the rods [9].

The focus of this paper is, however, not on the phase behavior as many previous studies, but on the structure of the fluid, in particular, the single particle distribution and twobody distribution functions. The nematic phase is often characterized by the orientational distribution function (ODF) $f(\theta)$, which is a single-particle distribution function and describes the distribution of orientations of the particles about a preferred direction, i.e., director. The angle between the long molecular axis and the director is denoted by $\theta \in[0, \pi / 2]$. This function plays a key role in understanding the properties of nematic phases and many theoretical investigations rely on the explicit functional form of it. The nematic order parameter $S$ of a system is quantified by
PACS number(s): 64.70.Md, 61.30.Gd, 61.30.Eb, 61.20.Ja

where $P_{2}$ is the second Legendre polynomial. The orientational distribution function can be determined experimentally by various methods; one of them is based on diffraction experiments, which measures the Fourier transform of a twobody correlation function. It is interesting to investigate how a single-particle distribution can be obtained from measurements of the two-body correlations. If we consider $N$ identical particles with orientations given by the unit vectors $\{\hat{\omega}\}$, and $\rho(\vec{r} ; \hat{\omega})$ describes the internal (electron) density of each individual particle, then the scattered intensity of the total system is given by

$$
\begin{aligned}
I(\vec{q})= & \left\langle\sum_{i=1}^{N} \sum_{j=1}^{N} \iint d \vec{r} d \vec{r} \rho\left(\vec{r}-\vec{r}_{i} ; \hat{\omega}_{i}\right) \rho\left(\vec{r}-\vec{r}_{j} ; \hat{\omega}_{j}\right)\right. \\
& \times \exp [i \vec{q} \cdot(\vec{r}-\vec{r})]\rangle \\
= & \left\langle\sum_{i=1}^{N} \sum_{j=1}^{N} \exp \left[i \vec{q} \cdot\left(\vec{r}_{i}-\vec{r}_{j}\right)\right] \vartheta\left(\vec{q} ; \hat{\omega}_{i}\right) \vartheta\left(-\vec{q} ; \hat{\omega}_{j}\right)\right\rangle,
\end{aligned}
$$

with $\vartheta\left(\vec{q} ; \hat{\omega}_{i}\right)=\int d \vec{r} \rho\left(\vec{r} ; \hat{\omega}_{i}\right) \exp [i \vec{q} \cdot \vec{r}]$, the Fourier transform of the (electron) density distribution $\rho\left(\vec{r} ; \hat{\omega}_{i}\right)$ of particle $i$. Note that $\rho\left(\vec{r} ; \hat{\omega}_{i}\right)$ depends on the orientation of particle $i$. The total scattered intensity can be split into a part $F(\vec{q})$ that depends on a single-particle distribution function and a second part $S(\vec{q})$ that includes the spatial and orientational correlations,

$$
I(\vec{q})=N F(\vec{q}) S(\vec{q}) .
$$

The intraparticle scatter or form factor $F(\vec{q})$ is given by 

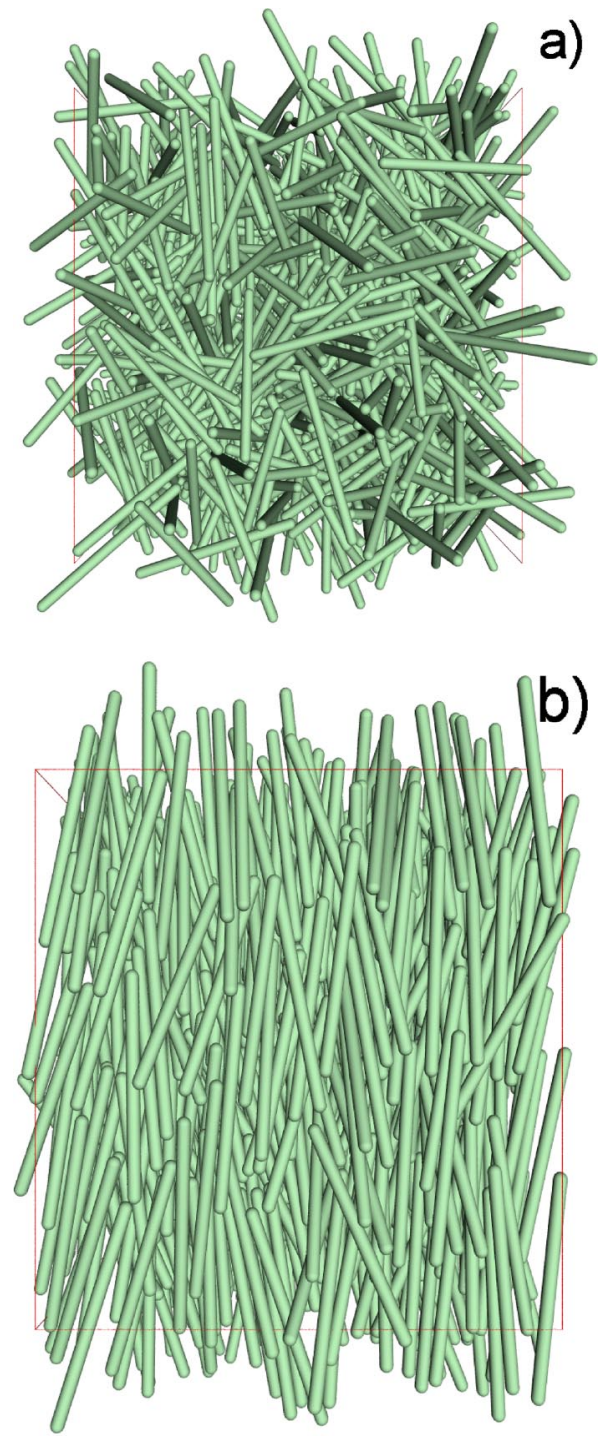

FIG. 1. Snapshot of a fluid of hard spherocylinders for packing fraction. (a) $\eta=0.1723$ (isotropic phase); and (b) $\eta=0.2338$ (nematic phase).

$$
F(\vec{q})=\left\langle|\vartheta(\vec{q} ; \hat{\omega})|^{2}\right\rangle,
$$

and the angular brackets denote an average over all the particles with their orientations given by the unit vectors $\{\hat{\omega}\}$. The interparticle scatter or structure factor term $S(\vec{q})$ reads [10] as

$S(\vec{q})=1+\frac{1}{N F(\vec{q})}\left\langle\sum_{i \neq j}^{N} \exp \left[i \vec{q} \cdot\left(\vec{r}_{i}-\vec{r}_{j}\right)\right] \vartheta\left(\vec{q} ; \hat{\omega}_{i}\right) \vartheta\left(-\vec{q} ; \hat{\omega}_{j}\right)\right\rangle$.

The structure factor depends on the positions of the rods and their relative orientations. For spherical symmetric particles, the (electron) density distribution $\rho(\vec{r} ; \hat{\omega})$ is independent of the orientation of the particle, and $\vartheta\left(\vec{q} ; \hat{\omega}_{i}\right)=\vartheta\left(\vec{q} ; \hat{\omega}_{j}\right)$. The structure factor is then decoupled from the form factor. However, for anisotropic density distributions, $\vartheta\left(\vec{q} ; \hat{\omega}_{i}\right)$ $\neq \vartheta\left(\vec{q} ; \hat{\omega}_{j}\right)$ and the structure factor is not decoupled from the form factor, hence, the structure factor includes spatial and orientational correlations. In the isotropic and nematic phase, it is often assumed that $S(\vec{q}) \rightarrow 1$ at a high angle and the intensity depends only on the form factor, i.e., $I(\vec{q})=F(\vec{q})$. If the intensity can be measured at a high angle, the orientational distribution function can be obtained directly from the intensity. At low angle, i.e., $|\vec{q}| D<2 \pi$, the scattering intensity depends on $S(\vec{q})$ which includes angular and spatial correlations between neighboring rods. In our simulations, we observe that $S(\vec{q}) \neq 1$ for $|\vec{q}| D<2 \pi$ and it is interesting to investigate the validity and consequences of the assumption $I(\vec{q}) \simeq F(\vec{q})$ that is often employed experimentally in the determination of $S$ and the ODF from intensity scatter. Leadbetter $[11,12]$ showed that the intensity distribution along the equatorial arc, thus at fixed $|\vec{q}|$, can be related to the ODF of the sample. The integral equation relating these two quantities was inverted by Deutsch [13]. However, using scattering data that includes experimental errors, a numerical inversion of the integral equation is very inaccurate. Therefore, one often assumes an analytical form of the ODF with some fitting parameters which are chosen such that it provides the best fit of the measured intensity distribution [14-16]. The resulting ODF and the nematic order parameter derived from the ODF may depend sensitively on the precise trial function of the ODF. The validity of these approaches to measure the nematic order and ODF in scattering experiments was studied by Purdy et al. [16] by comparing the nematic order parameter obtained from low angle scattering experiments (which include spatial and orientational correlations) with high angle scattering experiments (which depends only on the single-particle ODF). However, both approaches to obtain the nematic order parameter from low and high angle scattering involve approximations and are based on trial functions.

In this paper we test the validity of these approaches by determining the "exact" orientational distribution function of the rods, the "exact" nematic order parameter, and the intensity scatter directly in simulation. We compare the orientational distribution function and nematic order parameter obtained from the simulated intensity scatter using several trial functions with the "exact" results measured directly in our simulations.

The paper is organized as follows: In Sec. II we describe the simulations, in Sec. III we present the results, and the conclusions are drawn in Sec. IV.

\section{SIMULATIONS}

Extensive simulation studies are performed to determine the phase behavior of fluids consisting of hard spherocylinders [4-6,8,9]. The focus of this paper is, however, not on the phase behavior, but on the structure of the fluid, in particular, the single-particle distribution and two-body distribution functions. The "exact" orientational distribution function of the rods, the "exact" nematic order parameter, and the intensity scatter are determined directly in simulation. We study a system of hard spherocylinders in a cubic box with periodic boundary conditions. Each rod consists of a cylindrical part with diameter $D$ and length $L=15 D$ and of spheri- 
cal caps at both ends with diameter $D$. We present MonteCarlo simulations in the canonical ensemble, so we fix the number of particles at $N=1000$, and the volume $V$ of the system. We perform simulations at varying packing fractions $\eta=\pi\left(D^{3} / 6+D^{2} L / 4\right) N / V$ in the isotropic and nematic phase. In Fig. 1, we present snapshots of the isotropic and nematic phase at packing fractions $\eta=0.1723$ and 0.2338 , respectively. The packing fractions of the coexisting isotropic and nematic phases are predicted to be $\eta_{I}=0.1777$ and $\eta_{N}$ $=0.2058$, while the packing fraction of the nematic-smectic transition is at about $\eta_{S m} \simeq 0.4438$ [9].

The simulations are started from an initial condition in which the orientations of the rods are randomly distributed in a cone with $0 \leqslant \theta \leqslant 0.15 \pi$, where $\theta$ is the angle between the $z$-axis and the orientation of the rod. The deviations of the nematic director from the initial direction, which is along the $z$-axis, are often assumed to be small [17]. However, we find that during our simulations the director fluctuations have a significant impact on the value of the order parameter and moreover on the best fitting distribution of the ODF. We therefore determine for each configuration the actual nematic director and we determine the ODF and the nematic order parameter with respect to the present director. In this way we ignore the fluctuations of the nematic director, which can be achieved experimentally by switching on a magnetic field that fixes the nematic director of the sample. The current nematic director and the nematic order parameter are determined from the standard $3 \times 3$ nematic order parameter tensor defined as [18]

$$
Q_{\alpha \beta}=\frac{1}{N} \sum_{i=1}^{N} \frac{3 u_{\alpha}^{i} u_{\beta}^{i}-\delta_{\alpha \beta}}{2},
$$

where $u_{\alpha}^{i}$ is the $\alpha$-component of the unit orientation vector of particle $i$, and $\delta_{\alpha \beta}$ is the Kronecker delta. The nematic order parameter is given by the largest eigenvalue of this tensor and the corresponding eigenvector is the nematic director of the sample.

We check for equilibration by monitoring the nematic order parameter of the system. The maximum allowed values for displacement and rotational moves are chosen such that the product of the acceptance ratio and maximum displacement is maximum which corresponds with an acceptance ratio of about $25-30 \%$. When equilibrium is reached, we perform a production run of $1.5 \times 10^{6}$ sweeps (one displacement attempt per particle), while sampling is performed once every sweep. The quantities that are sampled are the nematic order parameter, the orientation distribution function $f(\theta)$, which is proportional to the probability to find a rod with an angle within $[\theta, \theta+d \theta]$ relative to the nematic director, and the scattered intensity $I(\vec{q})$ given by Eq. (3). It is convenient to write the reciprocal vector in cylindrical coordinates $\vec{q}$ $=\left(q_{r}, q_{z}, q_{\phi}\right)$. If the system possesses uniaxial symmetry relative to the nematic director lying along the $z$-axis, the scattered intensity depends only on $r$ and $z$ coordinates, and is independent of $\phi$.

The Fourier transform of the (electron) density of a spherocylinder $\vartheta(\vec{q} ; \hat{\omega})$ can be approximated by the Fourier transform of only the cylindrical part of length $L$ and diam- eter $D$ as the contribution from the caps is negligible for the given length-to-diameter ratio,

$$
\vartheta(\vec{q} ; \hat{\omega}) \sim j_{0}\left(\frac{L}{2} q_{z}\right) \frac{J_{1}\left(\frac{D}{2} q_{r}\right)}{\frac{D}{2} q_{r}},
$$

where $j_{0}(x)$ is the spherical Bessel function of the zeroth order, and $J_{1}(x)$ is the cylindrical Bessel function of the first order. Note that in Eq. (7) the polar axis of the coordinate system is defined along $\hat{\omega}$, which can easily be transformed to the coordinate system where the polar axis coincides with the direction of scattering vector $\vec{q}$. For infinitely thin rods, the Fourier transform is defined by $j_{0}(x)$ only and $J_{1}(x) / x$ allows for a finite diameter of the rods. The averaged square $\left\langle|\vartheta(\vec{q} ; \hat{\omega})|^{2}\right\rangle$ of this property is the formfactor $F(\vec{q})$ of the system that contains information about the shape, the inner structure of the molecule, and the ODF. The structure factor $S(\vec{q})$ of the system contains information about the positional and orientational correlations between the scattering particles. Equation (3) with (4) and (5), however, is not convenient for sampling the scattered intensity in simulations as it contains a double summation, and the difference $\vec{r}_{i}-\vec{r}_{j}$ in the exponent requires paying attention to the periodic boundary conditions. Using simple algebra the scattered intensity can be written as

$$
I(\vec{q})=\left(\sum_{i=1}^{N} \cos \left(\vec{q} \cdot \vec{r}_{i}\right) \vartheta\left(\vec{q} ; \hat{\omega}_{i}\right)\right)^{2}+\left(\sum_{i=1}^{N} \sin \left(\vec{q} \cdot \vec{r}_{i}\right) \vartheta\left(\vec{q} ; \hat{\omega}_{i}\right)\right)^{2},
$$

which does not contain double summation and allows for twice the higher resolution as the smallest nonzero $q_{\min }$ is given by $q_{\min }=2 \pi / L_{i}$ due to periodic boundary conditions compared to Eq. (3) where $q_{\min }=4 \pi / L_{i}$ with $L_{i}$ the dimension of the box in the $i$-th direction [due to the difference $\vec{r}_{i}-\vec{r}_{j}$ in Eq. (5)].

\section{RESULTS AND DISCUSSION}

As we wish to investigate whether correlations present in the interparticle scatter influences the determination of the ODF and $S$ from intensity scatter, we first determine the "exact" value of $S$ and the "exact" ODF of the rods directly in simulation. We perform simulations at varying packing fractions $\eta$ in the isotropic and nematic phase. We measure $S$ from Eq. (6), the ODF $f(\theta)$, and the scattered intensity $I(\vec{q})$. The latter two are both measured from simulations (i) where the $z$-axis is taken to be the fixed nematic director, i.e., nematic director fluctuations are included and (ii) where for each configuration the present nematic director is calculated such that nematic director fluctuations are disregarded. The latter can be achieved experimentally by applying a magnetic field that fixes the nematic director of the sample. We present results for the case that nematic director fluctuations are disregarded, unless stated differently. Table I shows $S$ as a function of $\eta$ for the statepoints we considered in this study. Note 
TABLE I. The nematic order parameter $S$ of a fluid of hard spherocylinders for different packing fractions $\eta$ determined directly in simulations ("exact"), determined from a fit of the "exact" orientational distribution function, and determined from a fit of the scattering intensity $I(\psi)$.

\begin{tabular}{lccccc}
\hline \hline \multicolumn{1}{c}{$\eta$} & 0.1846 & 0.1969 & 0.2092 & 0.2215 & 0.2338 \\
\hline "Exact" & 0.627 & 0.747 & 0.799 & 0.825 & 0.861 \\
Maier-Saupe fit of ODF & 0.631 & 0.754 & 0.805 & 0.824 & 0.866 \\
Onsager fit of ODF & 0.674 & 0.777 & 0.819 & 0.835 & 0.872 \\
Gaussian fit of ODF & 0.683 & 0.781 & 0.822 & 0.837 & 0.874 \\
Maier-Saupe fit of $I(\psi)$ & 0.568 & 0.708 & 0.762 & 0.775 & 0.832 \\
Gaussian fit of $I(\psi)$ & 0.596 & 0.732 & 0.779 & 0.790 & 0.840 \\
\hline \hline
\end{tabular}

that the statepoint corresponding with a packing fraction $\eta$ $\approx 0.185$ lies in the isotropic-nematic coexistence region. For this statepoint, we observe large fluctuations in the nematic order parameter during our simulations. Figures 2 and 3 show typical examples of the "exact" orientational distribution function $f(\theta)$.

The measured ODF's are fitted with three different trial functions containing one fitting parameter $\alpha$,

$$
\begin{gathered}
f_{1}(\theta)=N_{1}(\alpha) \exp \left(\alpha \cos ^{2} \theta\right), \\
f_{2}(\theta)=N_{2}(\alpha) \mathrm{e}^{-\theta^{2} / 2 \alpha^{2}}, \\
f_{3}(\theta)=\frac{\alpha}{\sinh \alpha} \cosh (\alpha \cos \theta),
\end{gathered}
$$

where $N_{i}(\alpha)$ are the normalization constants, such that $\int_{0}^{\pi / 2} d \theta \sin \theta f_{i}(\theta)=1$. The first ODF is the Maier-Saupe distribution, the second is the Gaussian distribution introduced by Odijk, and the third one was proposed by Onsager [3]. The trial function introduced by Oldenbourg [19] is not considered here as the fitting parameter can be chosen such that it coincides with the Maier-Saupe distribution. We determine the nematic order parameter $S$ from the three fitted ODF's

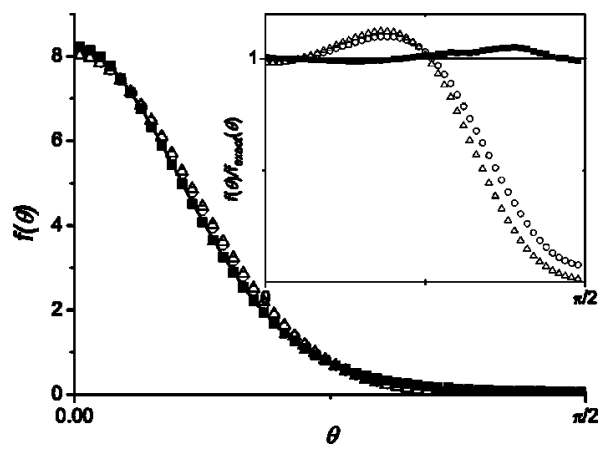

FIG. 2. Orientational distribution functions $f(\theta)$ for packing fraction $\eta=0.1846$. The solid line denotes the "exact" ODF measured directly in simulation. The "exact" ODF is fitted with the Maier-Saupe distribution ( $\square)$, the Onsager distribution ( ${ }^{\circ}$, and the Gaussian distribution $(\triangle)$. The inset shows the fits divided by the "exact" ODF.

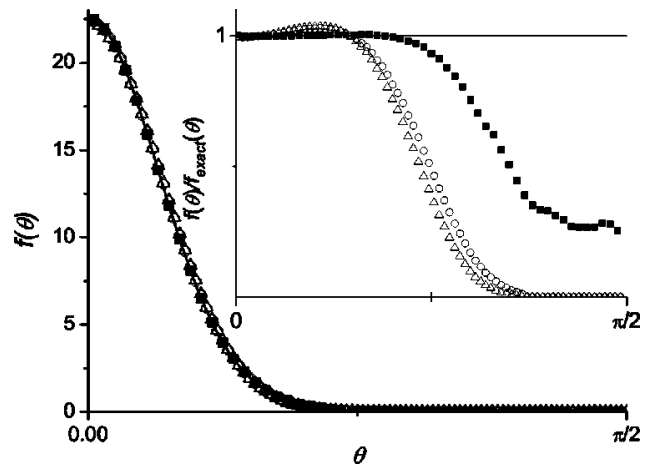

FIG. 3. Orientational distribution functions $f(\theta)$ for packing fraction $\eta=0.2338$. The solid line denotes the "exact" ODF measured directly in simulation. The "exact" ODF is fitted with the Maier-Saupe distribution $(\boldsymbol{\square})$, the Onsager distribution $(\bigcirc)$, and the Gaussian distribution $(\triangle)$. The inset shows the fits divided by the "exact" ODF.

using Eq. (1). The values of $S$ are listed in Table I and plotted in Fig. 4. Figures 2 and 3 and Table I show that at all densities the measured orientational distribution function is perfectly fitted by the Maier-Saupe distribution for all values of $\theta$ when nematic director fluctuations are ignored. The value of $S$ calculated with the fitted Maier-Saupe distribution matches closely to the one measured as the largest value of the nematic order parameter tensor (6). The fits of the ODF's using the Onsager and Gaussian distributions are less peaked then the measured ones, which result in a slightly overestimated nematic order parameter. However the nematic order parameters obtained from the different distributions differ only by a few percent. Increasing the density, the fits of the ODF's using different trial functions and the resulting values for $S$ approach each other.

We also study the influence of nematic director fluctuations on the ODF by comparing simulations where the $z$-axis is chosen to be the fixed nematic director with simulations where the nematic director is calculated for each configuration. As expected, we observe that the ODF is slightly

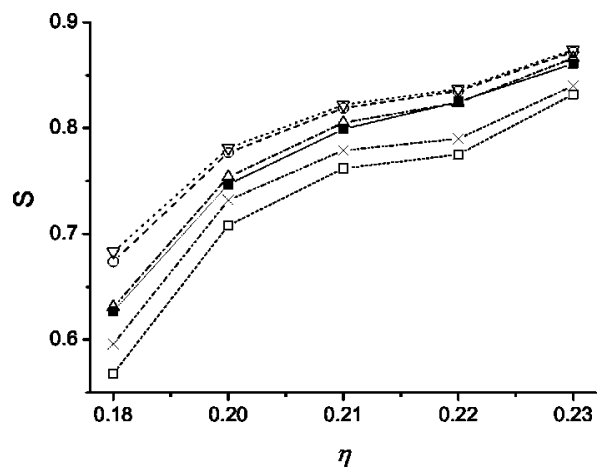

FIG. 4. The nematic order parameter $S$ as a function of the packing fraction $\eta$ obtained from different routes: direct measurement of (6) - - -, using a Maier-Saupe fit of the ODF $-\triangle-$, using a Gaussian distribution fit of the ODF $-\nabla-$, using an Onsager distribution fit of the ODF - - -, using an Onsager distribution fit of the intensity scatter $-X-$, using a Maier-Saupe fit of the intensity scatter $-\square-$. 
TABLE II. The nematic order parameter $S$ of a fluid of hard spherocylinders with packing fraction $\eta=0.2092$ determined directly in simulations with and without nematic director fluctuations.

\begin{tabular}{lcc}
\hline \hline Nematic director fluctuations & No & Yes \\
\hline "Exact" & 0.799 & 0.785 \\
Maier-Saupe fit of ODF & 0.805 & 0.769 \\
Gaussian fit of ODF & 0.822 & 0.793 \\
\hline \hline
\end{tabular}

broader and gives lower values of $S$ (see Table II) when we use the $z$-axis as the nematic director and, thus, allow for nematic director fluctuations. These findings are a logical consequence of the method we used for taking into account director fluctuations, i.e., via a reference to the fixed $z$-axis. More surprisingly, we find that the ODF is best fitted by the Gaussian distribution, when we allow for nematic director fluctuations, while the one without nematic director fluctuations is best fitted by the Maier-Saupe distribution. The values of $S$ are shown in Table II. In the sequel, we calculate the actual nematic director for each configuration and ignore nematic director fluctuations.

We plot the scattered intensity $I(\vec{q})$ in Fig. 5. As the simulated rods do not have any inner structure we do not observe a diffusive ring at high values of the scattering angle, and the scattered patterns resemble those obtained from interparticle scatter in experiments [16]. In experiments, the interparticle scatter is measured for $0 \leqslant|\vec{q}| D \leqslant 2 \pi$. We therefore focus ourselves to this range in simulations. Moreover, for $|\vec{q}| D$ $\gg 2 \pi$, the statistical accuracy of $I(\vec{q})$ decreases significantly as $F(\vec{q}) \rightarrow 0$ and the computational cost in measuring $I(\vec{q})$ increases dramatically. Figure 5(a) shows $I(\vec{q})$ for the isotropic phase at $\eta=0.1723$. We clearly see that $I(\vec{q})$ is isotropic and does only depend on $|\vec{q}|$. Figures 5(b)-5(d) show the intensity scatter for the nematic phase at varying $\eta$. We observe the typical anisotropic pattern of the scattering as also found experimentally for nematic solutions of the TMV and $f d$ virus $[10,16]$.

In order to obtain information about the spatial order of the system, we investigate the intensity scatter $I\left(q_{r}, q_{z}\right)$ along the equators $q_{z}=0$ and $q_{r}=0$. We plot $I\left(q_{r}, 0\right) / N, F\left(q_{r}, 0\right)$, and $I\left(0, q_{z}\right) / N$ in Fig. 6 for a nematic phase at $\eta=0.2092$ and $\eta=0.2338$. The inset shows $S\left(q_{r}, 0\right)$ which is obtained by dividing the intensity scatter per particle by the formfactor (3). The intensity at $|\vec{q}|=0$ is related to the isothermal compressibility $\chi_{T}$, i.e., $I(0,0)=N k_{B} T \chi_{T} / V$, where $k_{B}$ is Boltzmann's constant, $T$ the temperature, and $\chi_{T}=-1 / V(\partial V / \partial P)$ with $P$ the pressure. In the Gaussian approximation, the pressure of the nematic phase reads as $P \simeq 3 N k_{B} T / V$, yielding $I(0,0) \simeq 1 / 3$. Figure 6 shows indeed that $I\left(0, q_{z}\right) \simeq 1 / 3$ for $q_{z} \rightarrow 0$ and that $I\left(q_{r}, 0\right) \simeq 1 / 3$ for $q_{r} \rightarrow 0$. However, it is difficult to obtain an accurate estimate of the intensity $I\left(0, q_{z}\right)$ for $q_{z} \rightarrow 0$ as the intensity is a rapidly varying function of $q_{z}$ near the origin.

While the well-pronounced intensity peak as observed in $\mathrm{x}$-ray diffraction experiments of suspensions of a colloidal $\mathrm{fd}$ virus and TMV $[10,16]$ is missing in our $I(\vec{q})$, we do clearly observe a very broad peak in the structure factor, as found
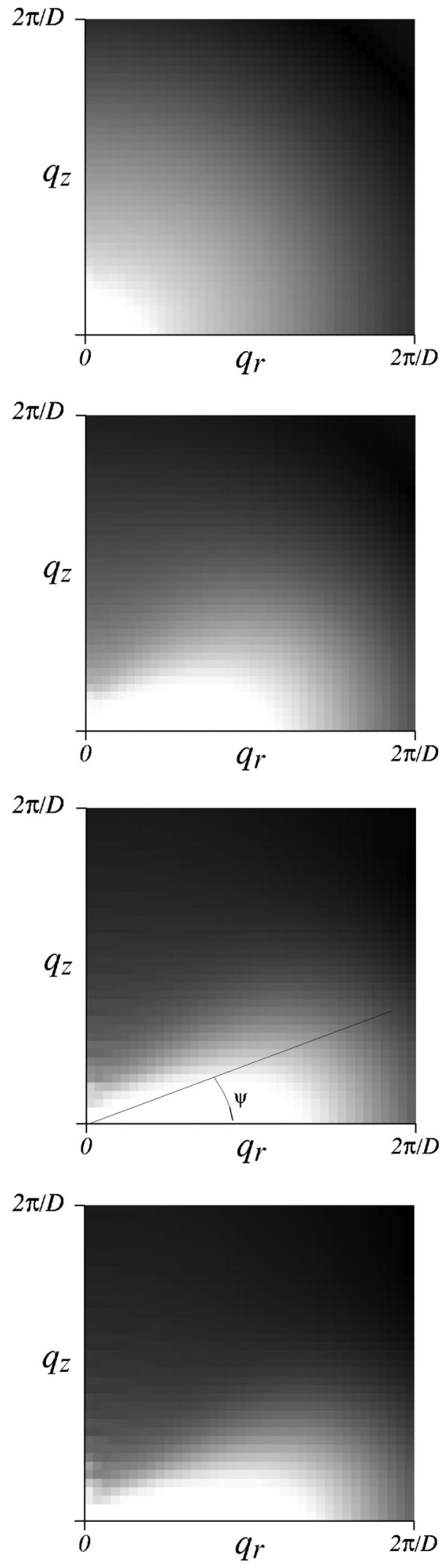

FIG. 5. Density plots of the intensity scatter $I\left(q_{r}, q_{z}\right)$ for varying packing fractions: (a) $\eta=0.1723$ (isotropic), (b) $\eta=0.1846$, (c) $\eta$ $=0.2092$, (d) $\eta=0.2338$. Bright areas correspond to high and dark areas correspond to low intensity scatter. 


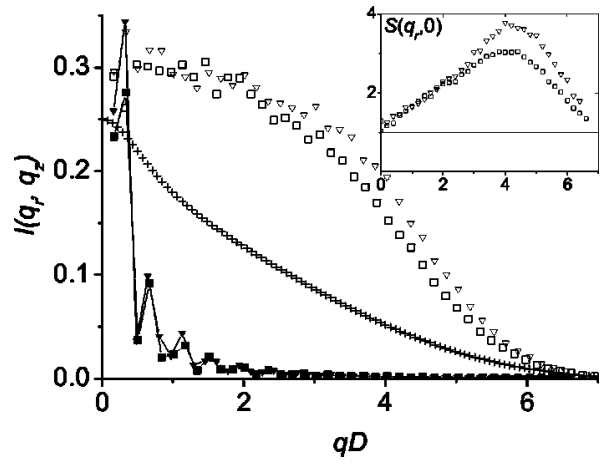

FIG. 6. Intensity distribution per particle along the $q_{r}=0$ and $q_{z}=0$ directions: $\square I\left(q_{r}, 0\right),-\square-I\left(0, q_{z}\right)$ for $\eta=0.2092, \nabla I\left(q_{r}, 0\right)$, $-\boldsymbol{\nabla}-I\left(0, q_{z}\right)$ for $\eta=0.2338$, together with the formfactor $F\left(q_{r}, 0\right)$ $(+)$, which is not significantly distinct for these two packing fractions. The inset shows the structure factor $S\left(q_{r}, 0\right)$ for the two packing fractions

experimentally. The main reason for the absence of the intensity peak is that the virus particles used in the experiments carry a surface charge and are dressed with a cloud of coand counterions, resulting in a much larger effective diameter $D_{\text {eff }}$ of the particles compared to the actual (scattering) diameter $D_{\text {scat }}$. Although the effective length-to-diameter ratio $L / D_{\text {eff }}$ of the $f d$ or TMV virus is close to our value for the length-to-diameter ratio $L / D$ of the simulated rods, the scattering length-to-diameter ratio $L / D_{\text {scat }}$ is much larger than $L / D_{\text {eff. }}$. If we use in our simulations of rods with $L / D=15$, the formfactor of infinitely thin rods (which is of course much broader than the one shown in Fig. 6 for finite $L / D$ ), we obtain an intensity peak similar to the one found in experiments. The diameters $D_{\text {eff }}$ and $D_{\text {scat }}$ in the simulations can be varied such that it matches the experiments. However, varying the diameters does not change significantly the normalized arc intensity distribution, and we therefore decided to use the same diameters for $D_{\text {eff }}$ and $D_{\text {scat }}$. The structure factor peak is at about $q_{r} D \sim 4$ corresponding with scattering from typical distances $2 \pi / q_{r} \sim 1.57 D$, which can be associated with the average rod separation in radial direction. We also observe that the structure factor peak moves to higher $q_{r}$ at increasing $\eta$ as expected. Compared to the strongly peaked structure factor peak in experiments of the colloidal $f d$ virus and TMV, we find a very broad structure factor peak in our simulations. Again, the discrepancy in the sharpness of the structure factor peak can be explained by the surface charge of the experimental rods, which increases the effective diameter of the rods considerable compared to the actual scattering diameter $D_{\text {scat }}$ in experiments. Moreover, we expect that the flexibility of the $f d$ virus particles broadens the structure factor peak compared to the rigid rods used in the simulations and counterbalances the effect of charge. It is worth noting that no structure factor peak was found by van der Schoot et al. [20] using infinitely thin rods, i.e., $D$ $=0$ and ignoring the cylindrical Bessel function of the first order in Eq. (7). An explanation is still lacking and it is interesting to study this in more detail.

We also find parallel to the nematic director presmectic correlation peaks in the intensity $I\left(0, q_{z}\right)$ at about $q_{z} D \sim 0.4$ corresponding with scattering from a typical distance $2 \pi / q_{z} \sim 16 D$ which is associated with the length of the rods. The appearance of presmectic peaks are in agreement with theoretical predictions by van der Schoot [21], who observed that the primary peak diverges at the spinodal instability to the smectic phase. It is worth noting that the presmectic peaks are already observed at packing fractions which are a factor of two smaller than the packing fraction at which the nematic-smectic phase transition occurs.

The intensity scatter in the direction of a vector $\vec{q}$ can be related to the orientational distribution function. At small absolute values of the scattering angle where the diffraction pattern depends on the structure factor one does not measure the single particle ODF but the coupled fluctuations of adjacent rods. This is expected to result in an overestimation of the order parameter $[11,22]$. At sufficiently high values of the scattering vector, it is often assumed that the structure factor approaches unity and the diffraction is determined by the form factor only. Note, however, that Fig. 6 shows that our structure factor does not approach unity for $q_{r} D<2 \pi$. Averaging over all possible orientations of the rods, we can approximate the scattering intensity to be

$$
I(\vec{q}) \simeq F(\vec{q})=\left\langle|\vartheta(\vec{q} ; \hat{\omega})|^{2}\right\rangle=\int d \Omega f(\Omega)\left|\vartheta\left(q_{r}(\Omega), q_{z}(\Omega)\right)\right|^{2}
$$

where $\Omega$ is the solid angle $(\theta, \varphi)$ of a rod with respect to the nematic director $(\theta)$ and azimuthally with respect to the incident beam $(\varphi)$. Due to the axial symmetry relatively to nematic director $f(\Omega)$ simplifies to $f(\theta)$. Using the explicit form of the Fourier transform (7) this expression can be easily related to the intensity distribution along the equatorial arc of diffraction pattern,

$$
\begin{aligned}
I(q, \psi)= & \int_{0}^{2 \pi} d \varphi \int_{0}^{\pi / 2} d \theta(\sin \theta) f(\theta) \\
& \times\left[j_{0}\left(\frac{L}{2} q \cos \alpha\right) \frac{J_{1}\left(\frac{D}{2} q \sin \alpha\right)}{\frac{D}{2} q \sin \alpha}\right],
\end{aligned}
$$

where $q$ is the absolute value of the scattering vector $\vec{q}$ along the arc, $\psi$ is the angle with the $q_{r}$-axis as denoted in Fig. 5, and $\alpha$ is defined by $\cos \alpha=\sin \psi \cos \theta+\cos \psi \sin \theta \cos \varphi$. This formula is difficult to use in the analysis of experimental data, and instead the expression relating $I(\psi)$ to $f(\theta)$ proposed by Leadbetter is commonly used [11,14],

$$
I(\psi)=\int_{\psi}^{\pi / 2} d \theta \frac{f(\theta) \sin \theta}{\cos ^{2} \psi \sqrt{\tan ^{2} \theta-\tan ^{2} \psi}} .
$$

It does not depend on the absolute value of the scattering vector and is based on several drastic assumptions which are discussed in $[14,22]$. Leadbetter proposed it for the intensity distribution along the "wide angle ring" corresponding to the 
lateral mean distance between neighboring molecules. An analytical inversion of this formula (12) was proposed by Deutsch [13] and reads as

$$
f(\theta)=-\frac{1}{N \sin \theta} \frac{d}{d \theta} \int_{\theta}^{\pi / 2} d \psi I(\psi) \frac{\tan \psi}{\sqrt{\tan ^{2} \psi-\tan ^{2} \theta}},
$$

with normalization constant $N=\int_{0}^{\pi / 2} f(\theta) \sin \theta d \theta$. This allows calculating the exact form of ODF from x-ray diffraction data. However, using scattering data that includes experimental and statistical errors, a numerical inversion of the integral equation is very inaccurate. Hence, one often employs a trial function for the ODF with some fitting parameters. If one uses the Maier-Saupe distribution function $f(\theta)=N(\alpha) \exp \left(\alpha \cos ^{2} \theta\right)$, the scattered intensity is described by $[23,24]$

$$
I(\psi)=N_{1}(\alpha) \frac{\exp \left(\alpha \cos ^{2} \psi\right)}{\sqrt{\alpha} \cos \psi} \frac{\sqrt{\pi}}{2} \operatorname{erf}(\sqrt{\alpha} \cos \psi),
$$

with fitting parameter $\alpha$. If we use the Onsager distribution function $f(\theta)=\alpha \cosh (\alpha \cos \theta) / \sinh \alpha$, the integration of (12) yields [25]

$$
I(\psi)=\frac{\alpha}{\sinh \alpha}\left[1+\frac{\pi}{2} L_{1}(\alpha \cos \psi)\right],
$$

where $L_{1}(x)$ is the modified Struve function of the first order. Unfortunately, the Gaussian form for the ODF does not lead to a simple expression for the intensity distribution.

A comparison of different distribution functions is straightforward due to simple normalization rules. The Leadbetter expression provides similar normalization for scattered intensity; provided that an orientational distribution function is properly normalized we can integrate the intensity along the arc to obtain $\int_{0}^{\pi / 2} d \varphi I(\varphi)=\pi / 2$ which is independent of the particular form of the trial ODF.

We determine the ODF from the intensity distribution along the equatorial arc of the x-ray diffraction patterns for different absolute values of the scattering vector to check how strong it depends on $|\vec{q}|$. This can serve as a test of the applicability of the formula proposed by Leadbetter, Eq. (12), which is independent on $|\vec{q}|$. In Fig. 7, we plot equatorial intensity distributions for packing fractions $\eta=0.1846$ and $\eta=0.2338$ with $3.7<|\vec{q}| D<6.3$. We do not observe strong dependence on $|\vec{q}|$, which agrees with the assumption used in the formula proposed by Leadbetter. However, Fig. 6 shows that the structure factor only tends to approach unity at the highest value of $q_{r}$, i.e., $q_{r} D=2 \pi$, and the approximation $I(\vec{q}) \simeq F(\vec{q})$ is not valid.

We, however, do fit our measured intensities with the intensity distributions using the Maier-Saupe distribution (14) and the Onsager distribution (15) and the corresponding ODF's are compared with the ones measured directly in simulation in Figs. 8 and 9. Figures 8 and 9 show clearly that the ODF obtained from intensity scatter are less peaked than the "exact" ones. Using the ODF's from intensity scatter, we
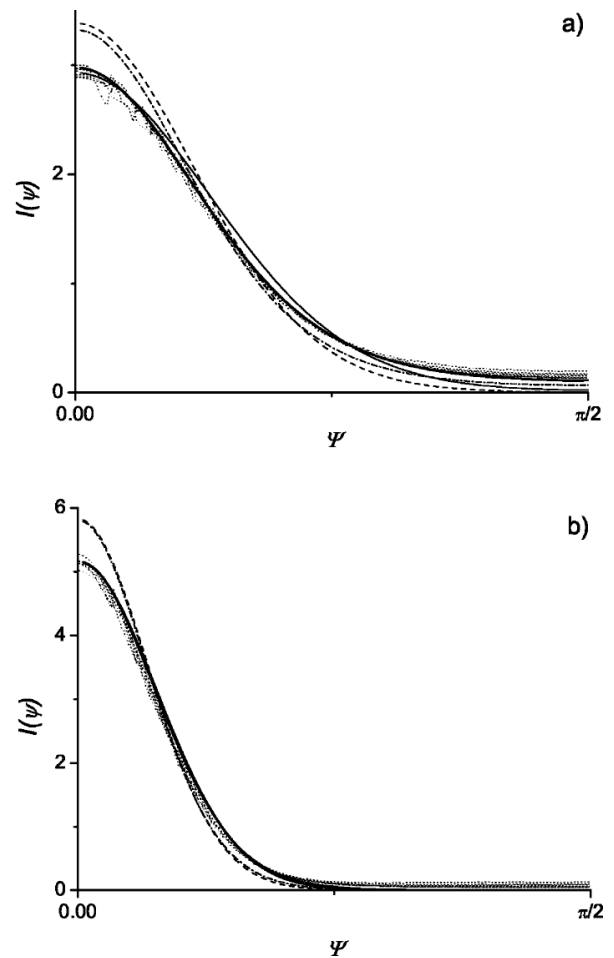

FIG. 7. Equatorial intensity distributions $I(\psi,|\vec{q}|)$ for packing fractions $\eta=0.1846, \eta=0.2329$, dots represent the intensities measured along several arcs with the absolute value of the scattering vector between $1.2 \pi<|q| D<2 \pi$; solid lines are fits of these intensities with distributions (14) (thick line) and (15) (thin line), dashed, and dashed-dotted lines are the intensities obtained from the formula proposed by the Leadbetter formula using the fits of the "exact" orientational distribution function with the Onsager and MaierSaupe distributions, respectively.

determine the nematic order parameter using (1). The values of the nematic order parameter obtained from intensity scatter underestimate the "exact" $S$ by 2-9\%. Although the "exact" ODF is best fitted by the Maier-Saupe distribution, the Onsager distribution gives a better estimate of the nematic order parameter calculated from intensity scatter as shown in Figs. 8 and 9. On the other hand, the result of the Leadbetter

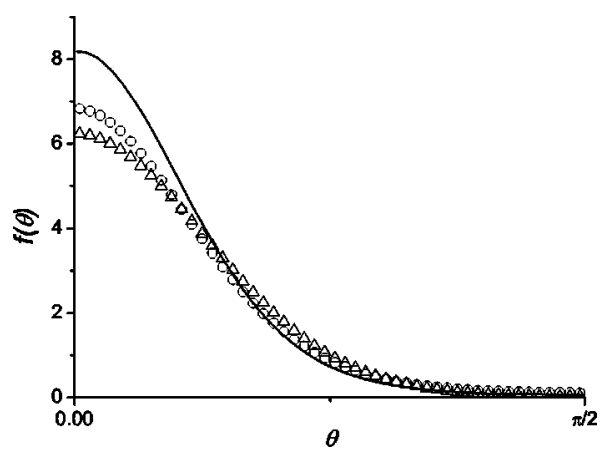

FIG. 8. Orientational distribution functions $f(\theta)$ for packing fraction $\eta=0.1846$. The solid line denotes the "exact" ODF measured directly in simulation. The intensity scatter is fitted with the Maier-Saupe distribution $(\bigcirc)$ and the Onsager distribution $(\triangle)$. 


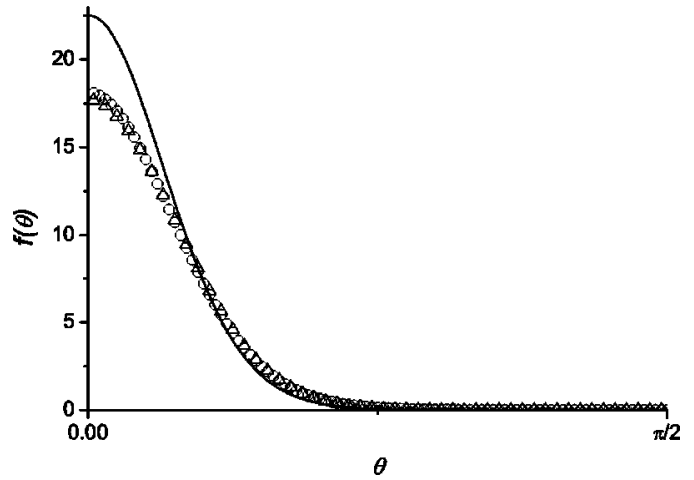

FIG. 9. Orientational distribution functions $f(\theta)$ for packing fraction $\eta=0.2338$. The solid line denotes the "exact" ODF measured directly in simulation. The intensity scatter is fitted with the Maier-Saupe distribution $(\bigcirc)$ and the Onsager distribution $(\triangle)$.

formula supplied with the fits using the Onsager and MaierSaupe distribution of the "exact" ODF is compared to the measured intensities in Fig. 7. Figure 7 shows, that the intensity distributions obtained from the fits of the "exact" ODF is more peaked than the measured intensity distributions.

\section{CONCLUSIONS}

The orientational distribution function, the nematic order parameter, and the intensity scatter are calculated in simulations for a system of hard spherocylinders with an aspect ratio of 15 for varying densities in the isotropic and nematic phase. The angular distribution function in the nematic phase is well described by the Maier-Saupe distribution when nematic director fluctuations are ignored. Including nematic director fluctuations, the ODF is best fitted by the Gaussian distribution. The Leadbetter approach is found to give a reasonable description of the x-ray scattering pattern, even when $S(\vec{q}) \neq 1$ and thus spatial and orientational correlations are present. The values of the nematic order parameter $S$ and the ODF's obtained from interparticle scattering were compared with the "exact" ones determined directly in simulation in order to study the effect of correlations between the rods. We find that the values for $S$ determined from interparticle scattering are smaller than the "exact" ones by about $2-9 \%$ and that the ODF's are broader than the "real" ones. We also find that the values for $S$ and the ODF are rather insensitive to the absolute value of the scattering vector which agrees well with the assumption proposed by Leadbetter that $I(q, \psi)$ along the equatorial arc does is dependent on $|\vec{q}|$.

The length-to-diameter ratio of the rods used in simulation are much smaller than those used in experiments on the colloidal $f d$ virus and TMV. However, the effective anisotropy can be tuned by the salt concentration such that it approaches the value used in simulation. It is interesting to investigate in more detail what the effect of anisotropy is on the intensity scatter. As already explained above, one expects that the structure factor peak becomes more pronounced along the $q_{r}$-direction upon increasing the aspect ratio of the rods. When the structure factor peak is sharper, one expects that $S(\vec{q})$ approaches 1 more rapidly and that the influence of the structure factor and correlations become less important in the determination of the nematic order parameter and the ODF from intensity scatter. One might also argue that a more pronounced structure factor peak as expected at higher aspect ratios of the rods may yield a peak in the intensity scatter as observed experimentally $[10,16]$, but which was never predicted theoretically [20]. This will be investigated in a future work.

\section{ACKNOWLEDGMENTS}

We thank Seth Fraden for stimulating discussions and we wish to thank the Dutch National Computer Facilities Foundation for access to the SGI Origin3800. The High Performance Computing group of Utrecht University is gratefully acknowledged for ample computer time.
[1] H. N. W. Lekkerkerker, P. Buining, J. Buitenhuis, G. J. Vroege, and A. Stroobants, NATO ASI series C, Vol. 460, in Observation, Prediction and Simulation of Phase Transitions in Complex Fluids (Kluwer Academic, Dordrecht, 1995).

[2] S. Fraden, NATO ASI series C, Vol. 460, in Ref. [1].

[3] L. Onsager, Ann. N.Y. Acad. Sci. 51, 627 (1949).

[4] D. Frenkel, J. Phys. Chem. 91, 4912 (1987).

[5] A. Stroobants, H. Lekkerkerker, and D. Frenkel, Phys. Rev. A 36, 2929 (1987).

[6] J. Veerman and D. Frenkel, Phys. Rev. A 41, 3237 (1990).

[7] D. Frenkel and B. Mulder, Mol. Phys. 55, 1171 (1985).

[8] D. Frenkel, H. Lekkerkerker, and A. Stroobants, Nature (London) 332, 822 (1988).

[9] P. Bolhuis and D. Frenkel, J. Chem. Phys. 106, 666 (1996).

[10] E. E. Maier, R. Krause, M. Deggelmann, M. Hagenbuchle, R. Weber, and S. Fraden, Macromolecules 25, 1125 (1992).
[11] A. Leadbetter and E. Norris, Mol. Phys. 38, 669 (1979).

[12] A. Leadbetter and P. Wrighton, J. Phys. (Paris), Colloq. 40, C3 (1979).

[13] M. Deutsch, Phys. Rev. A 44, 8264 (1991).

[14] P. Davidson, Prog. Polym. Sci. 21, 893 (1996).

[15] B. J. Lemaire, P. Pannie, J. Gabriel, and P. Davidson, Europhys. Lett. 59, 55 (2002).

[16] K. R. Purdy, Z. Dogic, S. Fraden, A. Ruhm, L. Lurio, and S. G. J. Mochrie, Phys. Rev. E 67, 031708 (2003).

[17] R. Kamien and G. Grest, Phys. Rev. E 55, 1197 (1997).

[18] A. Saupe, Z. Naturforsch. A 19A, 161 (1964).

[19] R. Oldenbourg, X. Wen, R. B. Meyer, and D. L. D. Caspar, Phys. Rev. Lett. 61, 1851 (1988).

[20] P. van der Schoot and T. Odijk, J. Chem. Phys. 93, 3580 (1990).

[21] P. van der Schoot, J. Chem. Phys. 117, 3537 (2002). 
[22] P. Davidson, D. Petermann, and A. M. Levelut, J. Phys. II 5, 113 (1995).

[23] A. Paranjpe and V. Kelkar, Mol. Cryst. Liq. Cryst. Lett. 102, 289 (1984)

[24] V. Kelkar and A. Paranjpe, Mol. Cryst. Liq. Cryst. Lett. 104,
139 (1987)

[25] A. P. Prudnikov, Y. A. Brychkov, and O. I. Marichev, Integrals and Series Vol. 2: Special Functions (Gordon and Breach, New York, 1990). 